\title{
Análisis perceptual del proceso de transferencia de conocimiento en maderas a instituciones en Costa Rica
}

\author{
Perceptual analysis of the process of transfer of knowledge \\ in woods to institutions in Costa Rica
}

\author{
Lupita Vargas-Fonseca $^{\circledR}{ }^{\circledR}$, Juan Carlos Valverde ${ }^{2 *}{ }^{\circledR}$, Diego Camacho-Cornejo ${ }^{1}{ }^{\circledR}$, \\ Cinthya Salas-Garita $^{1}{ }^{\circledR}$, Alexander Berrocal-Jiménez ${ }^{1}$
}

Vargas-Fonseca, L., Valverde, J. C., Camacho-Cornejo, D., Salas-Garita, C. y Berrocal-Jiménez, A. (2021). Análisis perceptual del proceso de transferencia de conocimiento en maderas a instituciones en Costa Rica. Colombia Forestal, 24(1), 17-30.

Recepción: 23 de septiembre 2019

\section{Resumen}

Se analizó de forma perceptual la transferencia de conocimiento en tecnología de la madera en instituciones educativas estatales de Costa Rica. Se realizaron capacitaciones activo-pasivas en temas de secado, preservado y calidad de la madera, llevando a cabo tres evaluaciones de aprendizaje (precurso, postcurso y 30 semanas luego del postcurso). Con estas se valuó la percepción, conocimiento adquirido y limitaciones de los sistemas empleados. Se obtuvo una gran similitud entre las temáticas de secado y preservado con conocimientos previos superiores al $65 \%$, aprendizajes del $85 \%$ y retenciones de conocimiento del $75 \%$; caso contrario se evidenció con la temática de calidad, la cual presentó un conocimiento previo del $20 \%$, aprendizaje del $83.5 \%$ y retención del $75.6 \%$; los temas de secado y preservado mostraron la mejor percepción, considerados como simples, aplicables, útiles y entendibles; mientras que el tema de calidad se consideró complicado, complejo, inaplicable pero útil. Finalmente, se encontró que el aprendizaje de secado y preservado debe ser una combinación de
Aprobación: 19 de septiembre 2020

$50 \%-50 \%$ entre teoría-práctica; mientras la calidad debería ser $25 \%$-75 \% teoría-práctica.

Palabras clave: educación activa, educación pasiva, aprendizaje, ciencias forestales.

\begin{abstract}
The transfer of knowledge in wood technology in state institutions of Costa Rica was perceptually analyzed. Conducting active-passive training in topics of drying, preserving and quality of wood, using three learning assessments (precursor, post-course and 30 weeks post-course); the perception, knowledge acquired and limitations of the systems used were evaluated. Obtaining a great similarity between the topics of drying and preserving with previous knowledge over $65 \%$, learning of $85 \%$ and knowledge retention of $75 \%$, different from the quality theme that presented a previous knowledge of $20 \%$, learning of $83.5 \%$ and $75.6 \%$ retention; The subjects of drying and preserved the best perception, considered as simple, applicable useful and understandable, while quality was considered complicated, complex, irrelevant but useful. Finally,
\end{abstract}

1 Escuela de Ingeniería Forestal, Tecnológico de Costa Rica. Cartago, Costa Rica.

2 Laboratorio de Ecofisiología Forestal y Aplicaciones Ecosistémicas (Ecoplant). Cartago, Costa Rica.

jcvalverde@tec.ac.cr. Autor de correspondencia. 
it was found that drying and preserving learning should be a 50-50 \% combination between theory and practice; while the quality should be $25-75 \%$ theory-practice.

Keywords: active education, passive education, learning, forestry.

\section{INTRODUCCIÓN}

En América Latina la madera se ha constituido como un material comúnmente usado para el desarrollo de edificaciones habitacionales, mueblería, estructuras recreativas, entre otros (García et al., 2019). Se trata de un material que se caracteriza por ser de fácil adquisición, con propiedades físicas y mecánicas que permiten tener una trabajabilidad amplia, con un costo accesible y adaptable a las condiciones climáticas de la región (Bakar y Kumar, 2019). Sin embargo, existe un desconocimiento generalizado sobre su correcto uso (Estriegana et al., 2019). Leal Filho et al. (2018) mencionan que la utilización exitosa de la madera en construcción se asocia al conocimiento del usuario en cuanto a las propiedades y características del material que adquiere, sus ventajas y desventajas y con ello define el uso óptimo del material.

El proceso de transferencia de conocimiento en tecnología de la madera generalmente ha sido limitado, se ha desarrollado de forma empírica, con conceptos generales y en algunos casos ambiguos (Sinakou et al., 2018), con sistemas de aprendizaje formados a partir de experiencias propias del usuario y con técnicas de prueba y error de resultados. El conocimiento técnico ha sido limitado, por lo general, para el consumidor final debido a un desconocimiento de tecnicismos, unidades de medida y practicidad de la información, lo que ha reducido el uso de la madera en múltiples áreas (Beverelli et al., 2017; Leal Filho et al., 2018), aspecto que es diferente en otros materiales más homogéneos como el concreto o el acero (Torkar y Krašovec, 2019). Por ello, es necesario el desarrollo de sistemas de aprendizaje que generen una transferencia eficiente de técnicas, tecnologías y elementos fundamentales de uso de la madera (Bakar y Kumar, 2019).

Los sistemas de aprendizaje habitualmente se han enfocado en dos líneas: pasivo y activo (Gutsch et al., 2019). El aprendizaje pasivo consiste en un proceso de transferencia teórico en el que el usuario desarrolla observaciones e hipótesis del conocimiento transmitido a través de ejemplos, explicaciones y teoría; pero, en el proceso, el conocimiento es completamente sistematizado en presentaciones, trabajos de revisión o discusiones (Torkar y Krašovec, 2019). En cambio, el aprendizaje activo es un proceso en el cual se desarroIlan prácticas, el usuario aprende conocimiento a través de experimentación, observación, interactuando con objetos y materiales que permitirán crear un entendimiento de los conceptos trasmitidos (Zazo-Muncharaz et al., 2015; Estriegana et al., 2019). Las tendencias actuales de aprendizaje buscan establecer un vínculo entre el aprendizaje activo y el pasivo, con el fin de que el usuario se empodere del conocimiento, pueda utilizarlo y adaptarlo a situaciones reales, creando con ello receptibilidad neurológica, capacidad de asimilación, conciencia de entendimiento y capacidad de retención de información (Camacho-Cornejo et al., 2017).

La transferencia de conocimientos en ciencias forestales presenta una serie de retos y limitaciones en su proceso de formación. Aspectos como el grado de educación, capacidad receptiva a la retroalimentación y corrección, así como interés y preconcepción previa del usuario a ser capacitado limitan en muchos casos que la transferencia sea eficiente y efectiva (Gutsch et al., 2019). En un estudio desarrollado por Post et al. (2019) sobre procesos de educación en manejo sostenible se identificó que la falta de interés previa a la capacitación en conjunto con pocas habilidades blandas para el trabajo en equipo se convirtió en la mayor limitación en el éxito de la transferencia de conocimientos. Además, la retención 
de conocimiento disminuyó por dificultades del equipo de trabajo en cuanto a motivación y experiencias previas. Por su parte, Neroni et al. (2019) denotan que la precepción previa en temas de viverización y manejo de especies tiende a disminuir la viabilidad del material y con ello se generan pérdidas económicas, por lo que es necesario disponer de conocimientos previos sobre habilidades y tipo de aprendizaje óptimo de los grupos a capacitar.

En el caso específico de instituciones del Estado, el proceso de formación y transferencia en tecnología de la madera es esencial como método para estimular la demanda de la madera en este sector. Beverelli et al. (2017) indican que la demanda de madera en la parte gubernamental es fundamental para procesos de mitigación de emisiones de $\mathrm{CO}_{2}$, estimulación del mercado interno y sustentabilidad el desarrollo constructivo. Por lo cual, el presente estudio tuvo como objetivo analizar perceptualmente la transferencia y eficiencia de conocimiento en tecnología de la madera a instituciones estatales en Costa Rica.

\section{MATERIALES Y MÉTODOS}

\section{Temáticas desarrollados y grupo de estudio}

Se abordaron tres temáticas de aprendizaje que fueron definidas por Silva et al. (2011): secado, definido por el proceso en el cual se reduce el contenido de humedad de la madera; preservado (definido como el proceso en que se aplican químicos para aumentar la durabilidad del material en el tiempo); y normas de calidad de la madera (Inte C98:2011), terminología de maderas (Inte 04-07-02-2014, 2014 e Inte C99:2014), para madera aserrada para uso general (INTE 06-0701-2011, 2011). Se utilizaron como ejemplos 10 especies forestales (Acacia mangium Willd., Alnus acuminata Kunth., Pochota fendleri (Seem.) W.S. Alverson \& M.C. Duarte., Cupressus lusitánica
Mill., Gmelina arborea Roxb., Swietenia macrophylla King, Tectona grandis L. f., Terminalia amazonia (J.F. Gmel.) Exell, Terminalia oblonga (Ruiz \& Pav.) Steud. y Vochysia guatemalensis Donn. Sm.). Estas son usadas actualmente en proyectos de reforestación comercial, también son tenidas en cuenta en el manejo de bosques naturales y en el desarrollo de productos maderables. En las temáticas se abarcaron aspectos como los tipos de secado y preservado existentes, aplicabilidad de la normativa, sus características, ventajas y desventajas; además de la existencia de normas que los evalúen y como se ponen en funcionamiento estas en sistemas productivos.

El proceso de aprendizaje se realizó con tres proveedurías de instituciones estatales del país y se dirigió a grupos con un promedio de 15 participantes, con edades entre los 25 a 50 años, de ambos géneros, con formación en ingeniería, arquitectura, construcción, administración o afines, con conocimiento mínimo en ciencias forestales o de implementación de la madera en usos múltiples. El proceso de selección de dichos participantes se dio en función de su disposición a participar en todas las actividades del curso y estar ligados en actividades laborales que vincularan los conocimientos en los temas.

\section{Proceso de aprendizaje implementado}

Se puso en marcha un sistema de aprendizaje de tipo significativo-observacional (Berasategi et al., 2016), el cual consistió en dos etapas: la primera de aprendizaje pasivo, enfocada en la impartición de clases magistrales sobre terminologías y conceptos básicos en los temas de secado, preservado de madera y calidad visual de la madera. La segunda fase se fundamentó en el desarrollo de aprendizaje activo, conformado por actividades grupales prácticas en las que se aplicaba el proceso de evaluación y operación del secado y preservado de la madera, así como una guía supervisada del control y evaluación de la calidad de la madera. 


\section{Análisis perceptual}

El análisis de percepción de cada una de las tres temáticas analizadas se desarrolló con parejas de adjetivos opuestos. Los adjetivos calificativos se seleccionaron con el fin de entender el grado de evolución en conocimiento de cada temática y, a su vez, dimensionar los puntos de mejora y elementos que deben ser replanteados. Las parejas de adjetivos se ubicaron de forma indirecta en las preguntas pre, post y luego de 30 semanas post curso. Las parejas de adjetivos fueron: simple-complejo, interesante-aburrido, entendible-inentendible, útil-inútil, aplicable-inaplicable, práctico-complicado, explicable-inexplicable. En cada encuesta se realizaron cuatro preguntas en las que estuvieron implícitas las parejas de adjetivos, con el fin de tener una muestra significativa durante el estudio.

\section{Evaluación del aprendizaje}

Se desarrolló en tres fases: la primera se aplicó previo a la impartición del conocimiento de cada una de las temáticas; la segunda se realizó una vez implementada la lección activo-pasiva de cada tema; y la tercera se desarrolló 30 semanas después de la finalización el curso. Estas evaluaciones se hicieron al mismo grupo de estudio y los aspectos considerados fueron:

- I) Conocimiento básico del tema: conocimiento de definiciones y conceptos base, utilidad de definiciones en licitaciones e impacto de la temática en la aplicabilidad de la madera.

- II) Aplicación real de las temáticas en las actividades laborales: entendimiento práctico de conceptos, relación de conocimientos para explicar casos y limitaciones de los tecnicismos para la toma de decisiones.

- III) Facilidad de obtención de conocimiento de las temáticas por cuenta propia: rangos de aceptabilidad de maderas, fuentes de comparación de datos y fuentes de consulta de aclaración de dudas o información extra.
- IV) Consideración de relevancia de la temática en el proceso de desarrollo de licitaciones: elementos que se consideran importantes tener en cuenta en la actividad laboral, variables desconocidas que cree son útiles e impacto de la mejora en la licitación con el conocimiento adquirido (CA).

- V) Consideración del grado de dificultad de la temática: problemas de entendimiento, limitaciones de aplicabilidad y dificultad para trasmitir conocimiento a segundas personas.

- VI) Interés de aprender las temáticas para uso en licitaciones: empatía con el CA, posibilidad de aplicar el nuevo conocimiento en las actividades laborales y posibilidad de aumentar el CA por interés personal.

\section{Análisis estadístico}

En primer lugar, se hizo una caracterización previa del curso a partir de un análisis descriptivo de conocimientos y habilidades prácticas de la temática, interés de aprendizaje y percepción de dificultad. Para definir si existía significancia en los resultados se realizó un análisis de varianza de una vía (Andeva), en el caso de presentar diferencias significativas en los resultados se aplicó la prueba de Tukey. La estimación del CA se desarrolló con los datos adquiridos del precurso, postcurso y 30 semanas luego del post curso, con lo que se generó la ecuación 1 (Camacho-Cornejo et al., 2017) en la cual conforme el valor del CA tienda a 100 la información cuantificable asimilada es mayor.

$$
C A(\%)=\left(\left(V C_{\text {Pas }}+V C_{A c t}\right) / 2\right) \cdot 100
$$

donde, $\mathrm{CA}$ es el conocimiento adquirido (\%), $\mathrm{VC}_{\text {Pas }}$ es el valor de conocimiento pasivo y $\mathrm{VC}_{\text {Act }}$ es el valor de conocimiento activo.

Los valores de VC activo y pasivo se generaron a partir de la ecuación 2, la cual se obtuvo de estudios previos de Camacho-Cornejo et al. (2017) con productores maderables de Costa Rica. 
$V C_{\text {Act } / \text { Pas }}=C P * 0.1+A A_{\frac{A c t}{\text { Pas }}} * 0.25+C R C_{\frac{A c t}{\text { Pas }}} * 0.15$ (2)

donde, VC es el valor de conocimiento activo o pasivo, CP es el conocimiento previo, AA es el aprendizaje adquirido activo o pasivo y CRC es la capacidad de racionamiento adquirido (conocimiento realmente aprendido).

La determinación de las curvas de capacidad teórica de retención (CTR) de aprendizaje en función del porcentaje de información suministrada (tanto activa como pasiva) se realizó en la encuesta postcurso, pues se consideró un momento oportuno para la evaluación por lo recién de la transferencia del conocimiento. En la encuesta se aplicaron preguntas cuyo fin fue entender cuánta era la capacidad máxima de asimilación del conocimiento teórico y práctico y con ello definir los puntos de aprendizaje máximo de cada temática. Para ello se realizó la prueba no paramétrica de Siegel-Tukey para comparación de dos paquetes de información y definir el grado de similitud.

Se procuró que el análisis perceptual de los cursos fuese no paramétrico. A cada una de las ocho parejas de adjetivos evaluados pre, post y 30 semanas luego del post curso se les aplicó la prueba de Kuiper para determinar el grado de distribución y relación entre los valores estimados en cada adjetivo y así estimar las diferencias entre las tres etapas. Además, se realizó un análisis tipo Sann (Analysis/ Deployment) a partir del principio de clasificación de VC activo y pasivo; de igual modo, se simuló en combinaciones de 0-100 \%, 25-75\%, 50-50\%, $75-25 \%$ y $100-0 \%$ (aprendizaje activo-pasivo, respectivamente), con ello se generaron matrices de funcionalidad, dificultad y retención del conocimiento que definiera la mezcla óptima de conocimiento teórico-práctico de cada temática evaluada.

Finalmente, a todas las encuestas se les aplicó un análisis de Cronbach para determinar la fiabilidad y eliminar las preguntas que generaron datos erróneos o atípicos, siendo el valor mínimo de aceptación 0.80. Los análisis estadísticos se realizaron con los programas Statistica versión 3.1
(Statsoft, 2015), con un nivel de significancia de 0.05 .

\section{RESULTADOS}

\section{Caracterización previa del grupo al proceso de aprendizaje}

La caracterización previa de los grupos de estudio (tabla 1) mostró conocimientos distintos en cada una de las temáticas analizadas. Se identificaron dos agrupaciones de similitud, la primera conformada por los temas de secado y preservado, y una segunda por la norma de calidad. Las variables de conocimiento básico del tema, aplicación de las temáticas y facilidad para conseguir información que disponía el grupo fue significativamente mayor con secado y preservado (superior al $55 \%$ ), comparado con el conocimiento en la norma de calidad que fue menor al 20.5 \%. El grupo de estudio prácticamente no contó con conocimiento suficiente sobre aspectos básicos, aplicación y consecución de información de normas de calidad de la madera; un tema el cual es considerado como totalmente nuevo.

De igual manera, con las variables de consideración de relevancia de la temática y grado de dificultad se encontró que el tema de normas de calidad presentó una predisposición distinta; mientras que el tema de secado y preservado se consideró significativamente más importante (promedio de $79.67 \%$ ) y significativamente menos complejo (promedio de $47.83 \%$ ), en comparación con la norma de calidad, la cual se consideró poco importante y complicado $(69.11 \%$ y $85.77 \%$, respectivamente). Finalmente, no se encontraron diferencias significativas entre los tres temas en cuanto a la concepción de interés de estas, siendo en promedio del $83.34 \%$.

\section{Análisis del CA y determinación de curvas de máximo aprendizaje}

Se determinó el CA (figura 1), el cual varió significativamente en cada temática desarrollada. 
Tabla 1. Valores medios de los parámetros considerados en la línea base de conocimientos del grupo de estudio aplicado en tres temáticas forestales al personal de instituciones estatales en Costa Rica

\begin{tabular}{ccc}
\hline Parámetro evaluado & Temática & Valor perceptual \\
\hline \multirow{2}{*}{ Conocimiento básico del tema } & Secado & $62.61^{\mathrm{a}}(9.44)$ \\
& Preservado & $58.50^{\mathrm{a}}(10.10)$ \\
& Norma de calidad & $20.20^{\mathrm{b}}(12.13)$ \\
\hline \multirow{2}{*}{ Aplicación real de las temáticas en las actividades laborales } & Secado & $55.80^{\mathrm{a}}(8.99)$ \\
Facilidad de obtención del conocimiento de las temáticas por cuenta & Preservado & $50.11^{\mathrm{a}}(9.15)$ \\
propia & Norma de calidad & $18.80^{\mathrm{b}}(10.88)$ \\
\hline \multirow{2}{*}{ Consideración de relevancia de la temática en el proceso de desarrollo } & Secado & $55.08^{\mathrm{a}}(8.22)$ \\
de licitaciones & Preservado & $62.44^{\mathrm{a}}(9.10)$ \\
& Norma de calidad & $16.88^{\mathrm{b}}(7.88)$ \\
\hline & Secado & $80.11^{\mathrm{a}}(10.01)$ \\
& Preservado & $79.22^{\mathrm{a}}(9.88)$ \\
& Norma de calidad & $69.11^{\mathrm{b}}(7.89)$ \\
\hline \multirow{2}{*}{ Consideración del grado de dificultad de la temática } & Secado & $48.99^{\mathrm{a}}(10.33)$ \\
& Preservado & $46.66^{\mathrm{a}}(12.22)$ \\
& Norma de calidad & $85.77^{\mathrm{b}}(9.00)$ \\
\hline
\end{tabular}

Valores entre paréntesis representa la desviación estándar; letras diferentes indican diferencias significativas con un $\alpha=0.05$.

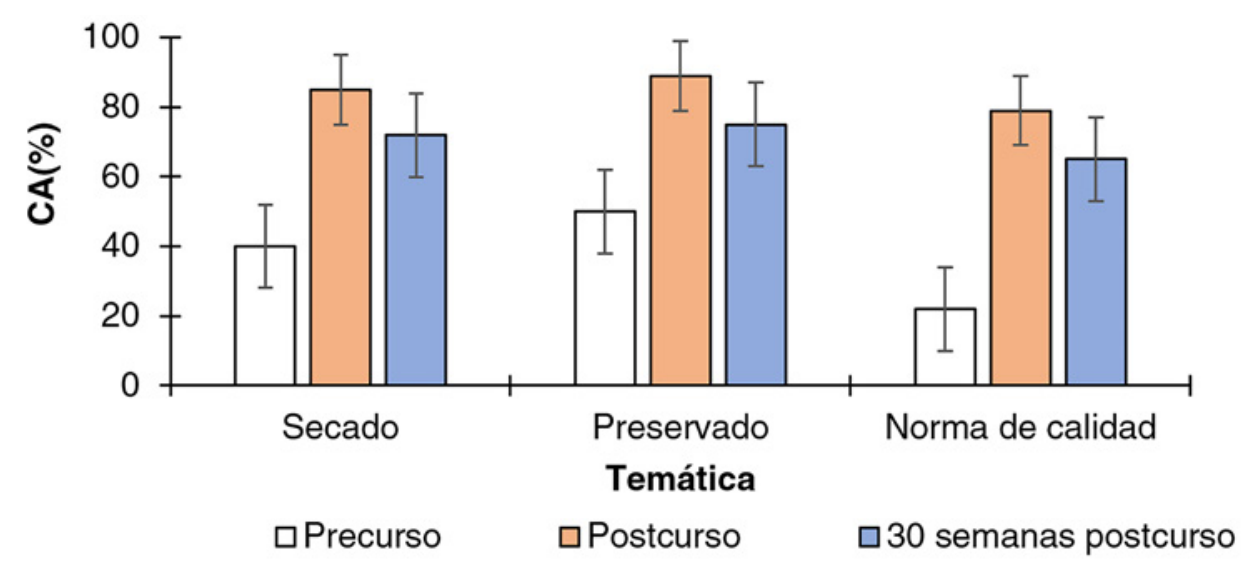

Figura 1. Conocimiento adquirido (CA) en la etapa de precurso, postcurso y 30 semanas luego del postcurso con tres temas de aprendizaje forestal a los grupos de personas que forman parte de instituciones estatales en Costa Rica.

Con el tema de secado el CA previo al curso fue significativamente menor $(40.0 \%)$ y aumentó a más del doble (82.5\%) posterior al curso, con una retención de conocimiento del 78.6 \%; la cual no mostró diferencias significativas con la evaluación postcurso, pues se obtuvo una retención de conocimiento elevada. Caso similar presentó la temática de preservado, el CA precurso fue del $43.5 \%$, posterior al curso se incrementó significativamente a un 87.6 \% con una retención en 30 semanas 
del $75.9 \%$; valor estadísticamente no significativo al obtenido en postcurso, siendo una retención de conocimiento elevada.

Finalmente, en relación con la calidad visual de la madera se evidenció un CA muy bajo, de apenas el $24.7 \%$, el cual una vez impartido el curso aumentó al 80.4 \%; pero, se presentó una disminución significativa de retención del 70.6 \%. Este es un valor moderado pero similar al obtenido por secado y preservado en el mismo periodo de tiempo.

Se determinaron las curvas teóricas de retención (CTR) de conocimiento en función del porcentaje de exposición de información de forma activa y pasiva (figura 2). Para la temática de secado (figura 2a) se obtuvo que la asimilación máxima de conocimiento activo es del $58.9 \%$, con un CTR máximo del $84.6 \%$. Sin embargo, posterior a este valor se presentó una pérdida de CTR producto de la incapacidad del grupo meta de asimilar de forma eficiente la información. En el caso del aprendizaje pasivo el punto máximo fue al $73 \%$ con un CTR de $78.8 \%$, mostrando para la temática de secado que el grupo de estudio presentó una capacidad de asimilar mayor cantidad información pasiva que activa. En el caso del tema de preservado (figura 2 b) el comportamiento varió, las curvas de aprendizaje activo y pasivo mostraron un comportamiento similar, el CTR aumentó conforme el porcentaje de exposición se acrecentó hasta el intervalo de 55.9 al $59.7 \%$, alcanzando un CRT máximo de $80.1 \%$ en aprendizaje activo y $77.1 \%$ en aprendizaje pasivo; posterior de dicho rango el CRT tendió a disminuir mostrando en la temática un aprendizaje balanceado entre ambas variables.

Finalmente, con el tema de caracterización visual de la madera (figura 2c) se obtuvieron valores de CRT máximo similares en aprendizaje activo y pasivo de $79.9 \%$, con la diferencia de que el porcentaje de exposición fue significativamente menor en el aprendizaje pasivo (punto máximo en $42.7 \%$ ) con respecto al aprendizaje activo (valor máximo de $57.3 \%$ ), siendo una temática en la cual el grupo de estudio mostró mayor adaptación al aprendizaje activo que al pasivo.

\section{Percepción del conocimiento obtenido durante el aprendizaje}

En la figura 3 se presenta la caracterización perceptual de los cursos pre, post y 30 semanas posteriores al curso. Se determinó de forma general las siguientes características:

I. La percepción de las tres temáticas mejoró significativamente posterior a la impartición de los cursos. Se consideraron los temas más simples, interesantes, entendibles, útiles y aplicables (figura 3a, 3b, 3c, 3d y 3f).

II. No se encontraron diferencias significativas en la percepción de practicidad y aplicabilidad (figura $3 g$ y $3 \mathrm{~h}$ ) de los temas entre el pre y postcurso (excepción en practicidad de secado), lo cual indica que se debe mejorar el proceso de transferencia de conocimiento de forma que el receptor tenga más clara la información.

III. La temática que mostró una mejora en percepción fue la de calidad visual de la madera, la cual mejoró hasta en un 62.2 \% con respecto a su concepción previa.

IV. La temática que mostró menor mejora de percepción fue la de secado, la cual apenas incrementó en un 38.9 \%.

V. Los calificativos de simplicidad, interés y entendimiento fueron los que mostraron mayor incremento, en promedio de un $52.6 \%$.

\section{Matriz de optimización de aprendizaje}

Producto de la caracterización perceptual y el análisis de la capacidad de retención de las tres temáticas se procedió a desarrollar una matriz de entendimiento de los modelos de aprendizaje (tabla 2). Se analizaron bajo los principios de la funcionalidad, grado de dificultad y capacidad de retención del conocimiento. Se obtuvo para la temática de secado que la mejor combinación funcional es de 50$50 \%$ o $25-75 \%$ entre aprendizaje pasivo y activo. 

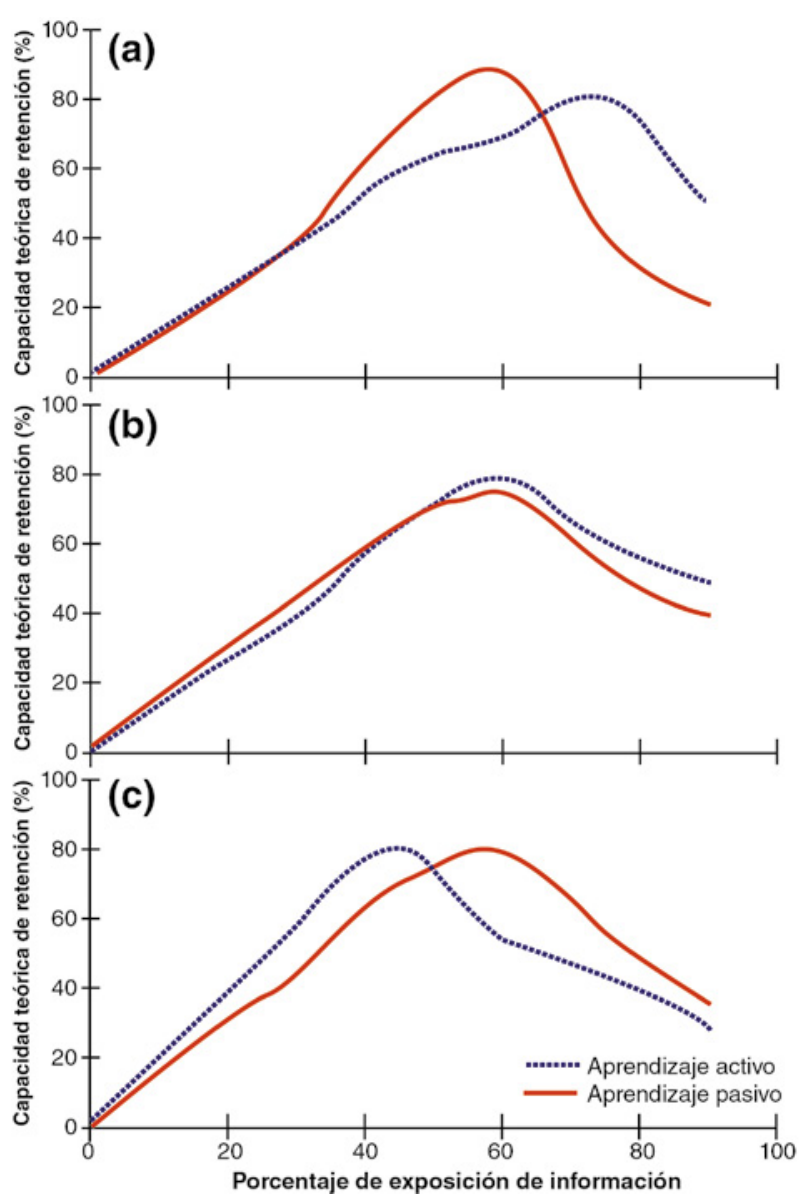

Figura 2. Capacidad teórica de retención en función del porcentaje de exposición de la información de tres temas de aprendizaje forestal a los grupos de personas que forman parte de instituciones estatales en Costa Rica.

Esta combinación es considerada como un sistema muy viable en el que se pueden dominar conceptos teóricos y entender su aplicación con casos reales en el desarrollo de licitaciones; pues, para dicha temática el realizar prácticas en que el usuario aprenda y entienda el impacto del secado es fundamental para que se mejoren las propiedades de la madera.

En el caso del tema de preservación la combinación 50-50 \% de aprendizaje pasivo-activo mostró mejores niveles de funcionalidad, menor impacto de dificultad y una capacidad de retención elevada; bajo este proceso equilibrado se generarían mejores índices de aprovechamiento de conocimiento.

Para la temática de calidad visual se determinó que la combinación de 25-75 \% de aprendizaje pasivo-activo mostraría mejores resultados, debido a la facilidad de entender el proceso mediante la aplicación del conocimiento teórico, en el cual el usuario pueda dimensionar la complejidad del tema, analizar casos y tener respuesta a situaciones reales de forma fluida. Esta combinación se mostró como la de mayor funcionalidad con un alto porcentaje de retención de conocimiento y con el menor grado de dificultad.

Finalmente, es importante destacar que cursos totalmente activos o pasivos que únicamente se enfoque en la práctica o teoría causarían una funcionalidad muy baja, la cual es debida a la omisión del contenido o limitación en la transferencia de criterios de análisis serían percibidos como complejos por la poca disponibilidad de información complementaria y presentarían una menor retención, lo que generaría inconsistencias y vacíos en el proceso de aprendizaje.

\section{DISCUSIÓN}

\section{Caracterización del aprendizaje}

Se encontraron diferencias significativas entre la temática de secado y preservado con respecto al tema de calidad visual de la madera. Ello se debe al grado de conocimiento previo que presentaron los grupos, pues para ambos temas era mayor en los grupos. Németh (2014) menciona que los conocimientos previos de una temática que es aprendida tienen influencia directamente en la capacidad de entendimiento y asimilación del conocimiento. Es decir, si el usuario cuenta con bases de conocimiento $y$, en especial, motivación para ampliar el conocimiento existente, la asimilación aumentará y con ello la retención de información será mejor. 


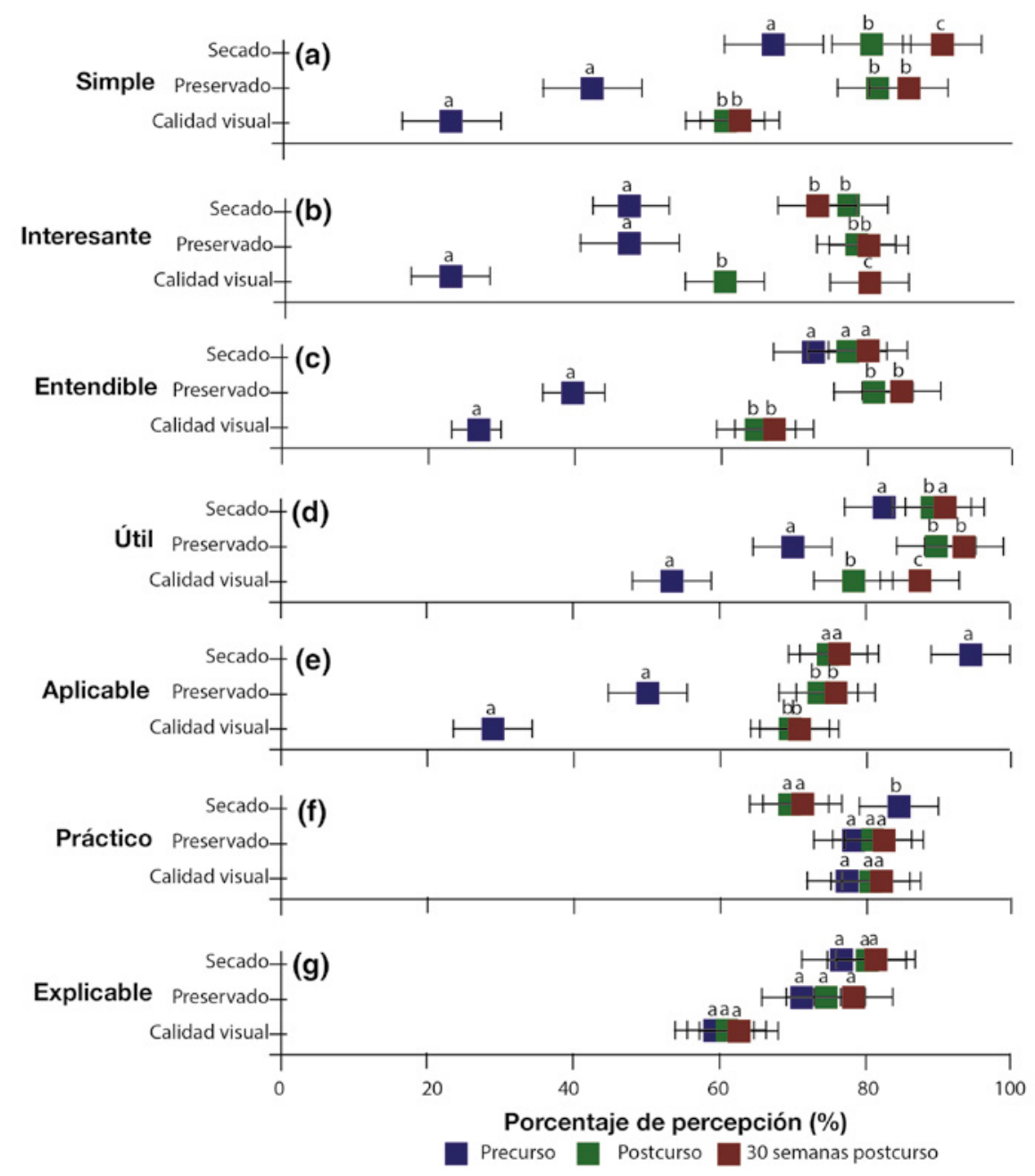

Figura 3. Caracterización perceptual en la etapa de precurso, postcurso y 30 semanas luego del postcurso con tres temas de aprendizaje forestal a tres grupos de estudio.

Tabla 2. Matriz de funcionalidad, dificultad y retención de conocimiento en combinación de teoría y práctica de tres temáticas de ciencias forestales

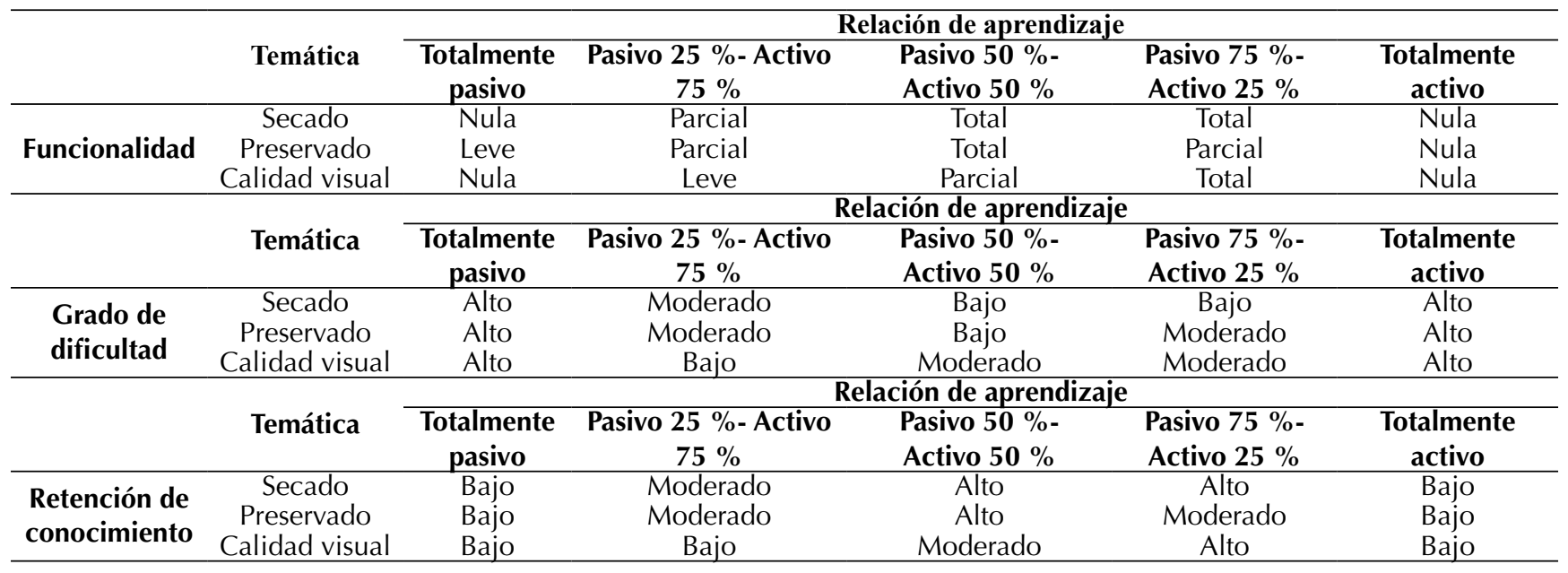


En el caso de la temática de calidad visual, la cual presentó un conocimiento menor, se evidenció una preconcepción de que sería una temática más compleja, pero con la aplicación de un sistema de aprendizaje activo se generó un aumento en el conocimiento y una retención superior al $70 \%$. Kashiwaba et al. (2012) remarcan que para temáticas nuevas en las cuales el usuario tenga percepciones negativas o de temor se debe transmitir el conocimiento de forma proactiva, de manera que el usuario se sienta cómodo, animado y empático con el conocimiento.

Con respecto a la capacidad de aprendizaje y retención del conocimiento, la aplicabilidad fue un factor de gran relevancia. McElwain et al. (2016) enfatizan en que un elemento clave para que el usuario aprenda y tenga empatía con la información es la capacidad de aplicarlo en sus actividades diarias. La constante reiteración de un concepto o elemento facilita la capacidad de retención de la información, por esta razón, en varias temáticas el aspecto práctico presentó mejores retenciones en comparación con la parte teórica; lo cual se evidenció en las curvas de CTR (figura 2). Al implementar en sus actividades laborales los temas de secado y preservado a los usuarios les resulta más fácil asimilar y reintegrar mejor la información práctica que los conceptos teóricos que son menos utilizados o, en algunos casos, dentro los carteles de licitación no se mencionan o explican a profundidad. Por su parte, Wahlgren et al. (2016) y Schendel y McCowan (2016) mencionan que en este tipo de actividades (en las cuales el elemento práctico tiene más reintegración que el elemento teórico) se debe promover en el usuario un análisis crítico de la actividad de forma consiente que reincorpore el concepto teórico y balancee la retención de información.

Otro factor que es mencionado por Kashiwaba et al. (2012) es la relación funcionalidad-tiempo. Este concepto se basa en la capacidad de desarrollar el CA en el tiempo (Prisặcariu, 2014), pues conforme el tema quese imparte sea funcional y aplicable(figura 3) el manejo de la temática será mejor, se disminuirá la tasa de error conceptual y presentará mayor fluidez neuronal en el momento de aplicar el conocimiento. La poca relación de funcionalidad-tiempo provoca que el conocimiento se pierda en el tiempo y su potencialidad de uso disminuya, lo cual ahonda la imposibilidad de mejorar la capacidad de retención. Cakula et al. (2015) destacan en este aspecto que temas complicados o de percepción compleja deben ser explotados en funcionalidad para mantenerse en el tiempo, de lo contrario la retención será mínima. Razón por la cual en el tema de la norma de calidad se muestra menores valores de aprendizaje, menor retención y mayor pérdida de retención y expectativa de aprendizaje. Además, el sistema empleado, en conjunto con su poca implementación laboral, genera funcionalidad baja en el tiempo; causando así el no ser recordado de forma adecuada por los usuarios. Por todo esto, la combinación activa-pasiva de $75-25 \%$ mejoraría la aplicabilidad y uso del conocimiento al momento de evaluar materiales de licitaciones o controles de calidad. Lo contrario se evidenció con los temas de secado y preservado de la madera, los cuales por su impacto y uso constante en sus actividades laborales tienden a ser funcionales en el tiempo.

\section{Efectos de la percepción en la capacidad de retención}

Las tres temáticas mostraron mejoría significativa de la percepción posterior a la realización de proceso de aprendizaje y 30 semanas luego del mismo. El incremento se debe al principio de corrección de preconcepciones (Neroni et al., 2019), en el que la explicación teórica complementada por prácticas, casos y ejemplos puede corregir la precognición o ambigüedades que existían hacia los temas por desconocimiento o implementación de fuentes de errores de conocimiento. Gutsch et al. (2019) destacan que la percepción negativa a procesos de aprendizaje se da por tres factores primordiales: 
I. Miedo, concepción de que el tema será complicado, complejo, difícil de aplicar y con alta exigencia de tiempo.

II. Fuentes erróneas de información, se da previo al aprendizaje a partir de conocimiento o experiencias de personas cercanas al usuario, lo cual incide en generar una preconcepción o inhibición de vivir lo mismo que las anteriores personas.

III. Poca capacidad de aprendizaje. El poco interés (combinado con limitaciones neuroquímicas y flexibilidad neuronal) imitan, o en el peor de los casos, impiden el correcto proceso de aprendizaje. Para lo cual, Estriegana et al. (2019) destacan que se deben desarrollar estrategias activo-pasivas en las que la percepción sea positiva, se estimule a resolver problemas de forma creativa, se maneje la presión en forma razonable y se dé un estímulo al usuario para continuar el aprendizaje posterior a la capacitación. De modo que, conforme mayor sea el dinamismo y menor la presión o regulación del conocimiento el aprendizaje se incrementará.

\section{Limitaciones del aprendizaje en conocimientos forestales}

Si bien las tres temáticas evaluadas presentaron comportamientos distintos (tabla 2), que se mostraron en los diferentes niveles de retención del conocimiento y en el impacto de la relación de aprendizaje activo y pasivo, también se evidencia un grado de similitud a nivel perceptual y se puede decir que son temas complementarios. Berasategi et al. (2016) destacan temas de aprendizaje paralelos que se puedan correlacionar en lecciones educativas que deben mostrarse mediante sistemas de aprendizaje neuronal, en los que se asocie cada contenido, se busque correlacionarlos y que el usurario los entienda en un solo conjunto articulado.

En la educación en temáticas forestales se debe evitar un desgaste cognitivo producto de procesos de educación estáticos, poco participativos o creativos, en los que los usuarios sientan que los conceptos o actividades realizadas son complicadas, poco útiles ni funcionales. En el caso de este proyecto los temas de secado, preservado y normas de calidad de la madera se presintieron como temas similares, por lo cual el sistema de aprendizaje usado fue funcional entre sí. Sin embargo, la percepción por parte de los usuarios no fue conforme, pues el tema de secado y preservado son paralelos por su común aplicabilidad en las actividades labores; en cambio, la norma de calidad no presenta el mismo patrón (figura 2) ya que mostró mayor complejidad y un conocimiento previo bajo, así como la necesidad de aumentar el aprendizaje pasivo más que el activo. García et al. (2019) mencionan que en estos casos se deben hacer modificaciones y analizar la carga de conceptos teóricos y prácticos, así como analizar las actividades prácticas desarrolladas y el manejo conceptual implementado; ya que temas con percepción variable no garantizan la utilización de los conceptos adquiridos a largo plazo y muestran pérdidas de retención superiores al $70 \%$.

Por tanto, es necesario hacer un análisis y replanteamiento de las técnicas empleadas con el fin de mejorar el aprendizaje y capacidad de retención del conocimiento, ya que los sistemas tradicionales no garantizan un efecto sustancial en el aumento del conocimiento a los grupos meta (Camacho-Cornejo et al., 2017). Zazo Muncharaz et al. (2015) comentan la necesidad de mejorar aspectos como: el reforzamiento extensionista, el manejo de conceptos claros, la paridad entre conceptos teóricos y prácticos, la eliminación de evaluaciones subjetivas y enfocarse en el desarrollo de sistemas de aprendizaje que permitan definir las fortalezas y debilidades del proceso empleado a partir de los cuales se pueda mejorar por medio de una constante actualización y reforzamiento de conceptos forestales adquiridos. Esto con el fin de mejorar la capacidad y retención del conocimiento de los usuarios meta en el tiempo. 


\section{CONCLUSIONES}

La aplicación del sistema de aprendizaje activo-pasivo en tres temáticas forestales mostró respuestas distintas. Con el tema de secado se presentó una mayor retención (superior al $65 \%$ ) y un desarrollo de aprendizaje, lo cual incidió en que el sistema puesto en marcha se interpretara como muy útil y simple. Caso similar ocurrió con la temática de preservado, la cual mostró un aprendizaje con una retención superior al 62.9 \% y una percepción final del tema como complicado pero muy útil. Estos resultados se debieron al conocimiento previo de los usuarios, en conjunto con la posibilidad de aplicación de conceptos en actividades diarias que incidieron en que estos conceptos sean funcionales en el tiempo.

Contrario a lo mencionado, la temática de norma de calidad presentó menor funcionalidad con el sistema de aprendizaje utilizado, lo que causó menor retención del conocimiento $(60.0 \%)$ y una percepción variable del curso debido a que su conocimiento previo fue bajo (al no aplicarse en sus labores diarias) y su funcionalidad no fue la óptima. Por ello, es necesario hacer revaloraciones o cambios en el sistema empleado para aumentar la retención y funcionalidad del tema.

Con este estudio se muestra la necesidad de analizar y adaptar el contenido forestal al usuario meta. Adicional a ello, que el conocimiento sea lo más claro, con la posibilidad de ser aplicado por el usuario y que pueda usarlo en sus actividades. Por último, se debe tener un control en el proceso de transferencia en el que se conozcan los puntos débiles de cada tema y, con ello, se desarrollen estrategias didácticas para mejorar la retención del conocimiento.

\section{AGRADECIMIENTOS}

Agradecemos a la Vicerrectoría de Investigación y Extensión (VIE), a la Unidad de Vinculación
Forestal y al Laboratorio de Ecofisiología Forestal y Aplicaciones Ecosistémicas (Ecoplant) por el apoyo logístico y presupuestario para la realización del proyecto.

\section{CONFLICTO DE INTERESES}

Los autores declaran no tener ningún conflicto de interés.

\section{CONTRIBUCIÓN DE CADA AUTOR}

L.V.F. contribuyó con la redacción de manuscrito. J.C.V. en la redacción manuscrito y análisis estadísticos. D.C.C., C.S.G. y A.B.J. con la impartición de conocimientos y realización de la evaluación de pruebas de conocimientos.

\section{REFERENCIAS}

Bakar, F. y Kumar, V. (2019). The use of humour in teaching and learning in higher education classrooms: Lecturers' perspectives. Journal of English for Academic Purposes, 40, 15-25. https://doi.org/10.1016/j.jeap.2019.04.006

Berasategi,N.,Alonso,N.y Roman,G. (2016).Service-learning and Higher Education: Evaluating Students Learning Process form their Own Percpective. Procedia-Social and Behavioral Sciences, 228, 424-429. https://doi.org/10.1016/j.sbspro.2016.07.065

Beverelli, C., Fiorini, M. y Hoekman, B. (2017). Services trade policy and manufacturing productivity: The role of institutions. Journal of International Economics, 104, 166-184. https://doi.org/10.1016/j.jinteco.2016.11.001

Cakula, A., Jakobsone, S. y Florea, M. (2015). Automated Learning Support System for Adult Education Institutions and Enterprises. Procedia Computer Science, 77, 191-198. https://doi.org/10.1016/j.procs.2015.12.383 
Camacho-Cornejo, L., Salas-Garita, C., Vargas-Fonseca, L. y Valverde-Otárola, J. (2017). Medición del conocimiento adquirido en tecnología de la madera por industriales forestales en Costa Rica. Tecnología en Marcha, 30, 35-46. https://doi.org/10.18845/tm.v30i3.3271

Estriegana, R., Medina-Merodio, J. y Barchino, R. (2019). Student acceptance of virtual laboratory and practical work: An extension of the technology acceptance model. Computers y Education, 40, 1-14. https://doi.org/10.1016/j.compedu.2019.02.010

García, E., Moizer, J., Wilkins, S. y Haddoud, M. (2019). Student learning in higher education through blogging in the classroom. Computers y Education, 136, 61-74. https://doi.org/10.1016/j.compedu.2019.03.011

Gutsch, M., Larondelle, N. y Haase, D. (2019). Of bugs and men: How forest pests and their management strategies are perceived by visitors of an urban forest. Urban Forestry y Urban Greening, 41, 248-254. https://doi.org/10.1016/j.ufug.2019.03.00

Inte 04-07-02-2014 (2014). Uso general de la madera Inte CTN 06. nteco.

Inte 06-07-01-2011 (2011). Madera estructural -Clasificación en grados estructurales para la madera aserrada mediante una evaluación visual. Inteco.

Kashiwaba, K., Kozawa, K. y Uchida, H. (2012). Evaluation of learning environment for ESD (Education for Sustainable Development) using activity index of salivary. Higher Education, 5, 1087-1090. https://doi.org/10.1007/978-94-007-3010-6_229

Leal Filho, W., Raath, S., Lazzarini, B., Vargas, V., de Souza, L., Anholon, R. y Klavins, M. (2018). The role of transformation in learning and education for sustainability. Journal of Cleaner Production, 199, 286-295. https://doi.org/10.1016/j.jclepro.2018.07.017

McElwain, A., Finnegan, V., Whittaker, A., Kerpelman, J., Adler-Baeder, F. y Duke, A. (2016). Evaluation and lessons earned from an undergraduate service learning course providing youth-focused relationship education. Evaluation and Program Planning, 58, 116-124. https://doi.org/10.1016/j.evalprogplan.2016.06.002
Németh, B. (2014). Research and Development of Adult Education through Higher Education Institutions: A Challenge and Perspective for Better Adult Learning and Education. Procedia-Social and Behavioral Sciences, 142, 97-103.

https://doi.org/ 10.1016/j.sbspro.2014.07.594

Neroni, J., Meijs, C., Gijselaers, J., Kirschner, P. y de Groot, R. (2019). Learning strategies and academic performance in distance education. Learning and Individual Differences, 73, 1-7. https://doi.org/10.1016/j.lindif.2019.04.007

Post, L., Guo, P., Saab, N. y Admiraal, W. (2019). Effects of remote labs on cognitive, behavioral, and affective learning outcomes in higher education. Computers y Education, 140, 1-19. https://doi.org/10.1016/j.compedu.2019.103596

Prisặcariu, A. (2014). Approaches of Quality Assurance Models on Adult Education Provisions. Procedia-Social and Behavioral Sciences, 142, 133-139. https://doi.org/10.1016/j.sbspro.2014.07.623

Schendel, R. y McCowan, T. (2016). Expanding higher education systems in low- and middle-income countries: the challenges of equity and quality. Higher Education, 72, 407-411. https://doi.org/10.1007/s10734-016-0028-6

Silva, W., Silva, L., Silva, C. y Nascimento, P. (2011). Optimization and simulation of drying processes using diffusion models: application to wood drying using forced air at low temperature. Wood Science and Technology, 45, 787-800. https://doi.org/10.1007/s00226-010-0391-x

Sinakou, E., Boeve-de Pauw, J., Goossens, M. y Van Petegem, P. (2018). Academics in the field of Education for Sustainable Development: Their conceptions of sustainable development. Journal of Cleaner Production, 184, 321-332. https://doi.org/10.1016/j.jclepro.2018.02.279

Statsoft (2015). Statistica, version 9.0. Londres: Statsoft. http://www.statsoft.com

Torkar, G. y Krašovec, U. (2019). Students' attitudes toward forest ecosystem services, knowledge about ecology, and direct experience 
with forests,. Ecosystem Services, 37, 1-19. https://doi.org/10.1016/j.ecoser.2019.100916

Wahlgren, B., Mariager-Anderson, K. y Sørensen, S. (2016). Expanding the traditional role of the adult education teacher-The development of relational competences and actions. Teaching and Teacher Education, 60, 303-311.

https://doi.org/10.1016/j.tate.2016.09.005
Zazo Muncharaz, J., de los Rios Carmenado, I. y Rivera, M. (2015). Education Planning Evolution for Forest Engineering in Spain. Procedia-Social and Behavioral Sciences, 197, 1710-1715. https://doi.org/10.1016/j.sbspro.2015.07.224 